\title{
Mehr Aktionärsrechte und weniger Interessenskonflikte für eine verbesserte Corporate Governance
}

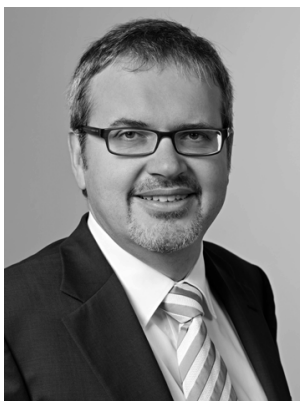

Christoph Kaserer

Corporate Governance, Aktionärsrechte, Interessenkonflikte, Board Primacy, Institutionelle Investoren

Corporate governance, shareholder rights, conflicts of interest, board primacy, institutional investors

Die Finanzmarktkrise hat wieder einmal gezeigt, dass es erhebliche Defizite in der Unternehmenskontrolle gibt. In diesem Beitrag wird argumentiert, dass neben einer Weiterentwicklung der Aktionärsrechte vor allem auch über Regelungen zur Begrenzung der Interessenskonflikte von institutionellen Investoren nachgedacht werden muss.

The financial crisis has once again shown the deficiency of our system of corporate control. In this article it is argued that for improving this system we need a twofold approach. First, shareholder rights have to be further developed. Second, a particular emphasis has to be put on rules reducing the conflicts of interest governing the behavior of institutional investors.

\section{Gibt es einen Trend zum Shareholder Empowerment?}

Es ist unumstritten, dass Defizite in der Unternehmenskontrolle eine Mitschuld an der Finanzmarktkrise hatten. In Verbindung mit der bereits seit längerem geführten Diskussion um eine exzessive Managerentlohnung ist in der Öffentlichkeit ein tiefes Misstrauen gegenüber den Corporate-Governance-Strukturen entstanden. Entsprechend sind verschiedene regulatorische Initiativen auf den Weg gebracht und teilweise auch schon umgesetzt worden. Stellvertretend sei das vom deutschen Bundestag verabschiedete Gesetz zur Angemessenheit der Vorstandsvergütung (VorstAG) ${ }^{1}$ genannt, durch welches sich die rechtlichen Rahmenbedingungen für Aktiengesellschaften bei der Festsetzung der Gesamtbezüge wesentlich geändert haben. Vorstandsbezüge müssen demnach einer Angemessenheitsprüfung unterzogen werden, variable Vergütungskomponenten müssen eine mehrjährige Bemessungsgrundlage haben und schliesslich können börsennotierte Gesellschaften ihre Vergütungssysteme einem fakultativen Hauptversammlungsbeschluss unterziehen (Say on Pay).

In ähnlicher Weise hat der US-amerikanische Gesetzgeber nach mehreren Anläufen und vorläufigen Regelungen mit Sec. 951 des Dodd-Frank Wall Street Reform and Consumer Protection Act 2010 den Securities Exchange Act um eine Section 14A erweitert, welche

1 BGBl. I 2009, $2509 \mathrm{ff}$. 
nun Say-on-Pay-Regelungen enthält. Konkret muss die Hauptversammlung einer amerikanischen börsennotierten Gesellschaft jetzt mindestens im Dreijahresrythmus über die Vorstandsvergütung, einschliesslich eventueller golden-parachute-Regelungen, entscheiden. Die Abstimmung ist bindend. Auch in verschiedenen anderen Ländern sind vergleichbare Regelungen eingeführt worden.

Grundsätzlich kann das Say-on-Pay-Beispiel als Beleg dafür genommen werden, dass es in der Corporate Governance weltweit einen Trend zur Stärkung der Aktionärsrechte gibt (shareholder empowerment). Dies gilt umso mehr, als es weitere Belege hierfür gibt. Genannt sei etwa die in den USA über ein Jahrzehnt lang geführte Debatte, ob und in welchem Umfang aussenstehenden Aktionärsgruppen über einen Zugang zu den von der Unternehmensverwaltung versendeten Stimmrechtsunterlagen (proxy document) die Möglichkeit eingeräumt werden soll, eigene Kandidaten für die Board-Wahl vorzuschlagen. ${ }^{2}$ Nach langem Hin und Her hat der für amerikanische börsennotierte Gesellschaften besonders wichtige Bundesstaat Delaware im Jahr 2009 eine entsprechende gesetzliche Regelung eingeführt. Im Jahr 2010 hat dann die SEC sogar noch eine verschärfte Regelung erlassen, mit der die Unternehmensverwaltung kaum noch eine Möglichkeit hatte, diesen Zugang $\mathrm{zu}$ verweigern.

Ein weiterer Beleg für den erwähnten Trend zur Stärkung der Aktionärsrechte ist die Debatte um die Managerhaftung. In den USA wurde bereits mit dem Sarbanes-Oxley Act 2002 eine Verschärfung eingeführt. In Deutschland wurde mit dem bereits erwähnten VorstAG ein verbindlicher Selbstbehalt bei Haftpflichtversicherungen von Vorständen in Höhe des 1,5-fachen des jährlichen Festgehalts gesetzlich festgeschrieben. Ausserdem wurde im Jahr 2010 \$ 93 Abs. 6 Aktiengesetz (AktG) dahingehend geändert, dass die Verjährungsfrist von Ansprüchen aus der Verletzung von Sorgfaltspflichten von Vorstandsmitgliedern bei börsennotierten Gesellschaften von 5 auf 10 Jahre erhöht wurde.

Trotz dieser Beispiele muss man allerdings auch darauf hinweisen, dass es auch Belege für einen in die gegenteilige Richtung weisenden Trend gibt, also einen Trend zur Stärkung der Rechte der Unternehmensverwaltung (board primacy). So muss man erwähnen, dass die oben beschriebene SEC-Regelung betreffend den Zugang zu Stimmrechtsunterlagen nach heftigstem Widerstand seitens der börsennotierten Gesellschaften im Juli 2011 durch ein amerikanisches Berufungsgericht wieder zu Fall gebracht wurde (Roe 2011, 12).

In Deutschland hat der Gesetzgeber auf die Angriffe von Hedge Fonds auf verschiedene börsennotierte Gesellschaften ${ }^{3}$ mit dem so genannten Risikobegrenzungsgesetz reagiert. ${ }^{4}$ Es sieht unter anderem vor, dass sich eine Gesellschaft auf der Grundlage eines Satzungsbeschlusses weigern kann, so genannte Nominees (Treuhänder) in das Aktienregister einzutragen. Unabhängig davon soll eine Gesellschaft zudem jederzeit das Recht haben, von einem eingetragenen Aktionär die Offenlegung des wahren Inhabers der Aktien zu verlangen; eine Auskunftsverweigerung führt zu einer Aussetzung des Stimmrechts. Angelehnt ist dieser Vorschlag an eine ähnlich ausgestaltete Eintragungsbeschränkung in der Schweiz. Wie man aus der Rechtspraxis der Schweiz weiss, stellt dieses Instrument eine relativ scharfe Waffe bei Streitigkeiten mit Aktionärsgruppen dar. Verschärft wird die Wirkung dieser Massnahme durch eine Neufassung der Regelungen zum acting in concert nach $\mathbb{2 2}$ Wertpapierhandelsgesetz (WpHG). Jetzt liegt ein abgestimmtes Verhalten von Aktionären

2 Für eine detaillierte Analyse dieser Entwicklung vgl. Roe (2011).

3 Besondere Aufmerksamkeit erregte der Fall der Deutschen Börse im Jahr 2005.

4 BGBl. I 2008, $1666 \mathrm{ff}$. 
bereits dann vor, wenn sie auf eine nicht näher definierte Weise mit dem Ziel „zusammenwirken “, die unternehmerische Ausrichtung der Gesellschaft erheblich zu beeinflussen. Da eine Zurechnung von Stimmrechtsanteilen gravierende Auswirkungen haben kann, man denke etwa an die Überschreitung der 30\%-Stimmrechtsanteilsgrenze, die ein Pflichtangebot auslöst, birgt diese Vorschrift gerade für institutionelle Investoren ein erhebliches Risikopotential.

Vor diesem Hintergrund stellt sich daher die Frage, ob es tatsächlich richtig ist, wenn verschiedene Autoren von einem Trend zum shareholder empowerment sprechen. Zweifellos lassen sich zahlreiche Belege dafür finden, gleichzeitig finden sich aber auch Belege für eine gegenteilige Entwicklung. ${ }^{5}$ Allerdings ist die Beantwortung dieser Frage auch nicht so entscheidend, denn die grundsätzliche Frage muss vielmehr lauten, welche regulatorischen Veränderungen für eine Verbesserung der Corporate Governance notwendig sind. Bevor man sich dabei auf die Diskussion einzelner Massnahmen einlässt, muss man sich in der Tat die Frage stellen, ob man eine Stärkung der Aktionärsrechte (und damit auch des Kapitalmarktes) oder eine Stärkung der Rechte der Unternehmensverwaltung als grundsätzliche Stossrichtung verfolgt.

Man kann sich dieser Frage sowohl unter theoretischen als auch empirischen Gesichtspunkten nähern. Auf Grund der gebotenen Kürze des hier vorliegenden Dialogbeitrags möchte ich mich auf den zweiten Aspekt konzentrieren. Dabei drängen sich zwei Fragestellungen auf. Einerseits kann man sich die Aktienkursreaktionen auf solche regulatorische Massnahmen anschauen, die den Einfluss von Aktionären in erheblicher Weise verändern. Andererseits kann man sich die Wirkungen von Kontrollaktivitäten von Aktionärsgruppen auch ganz direkt anschauen. Aufgrund der grossen Zahl einschlägiger empirischer Untersuchungen kann diese Vorgehensweise einen Eindruck darüber vermitteln, ob und ggf. in welcher Weise ein shareholder empowerment eine Verbesserung der Corporate Governance erwarten lässt.

\section{Die Wirkung der Kontrollaktivitäten von Aktionärsgruppen}

Wie von Grossman/Hart (1980) schon vor vielen Jahren treffend beschrieben, leidet die Effektivität der Unternehmenskontrolle durch Streubesitzaktionäre unter einem free-riderProblem. Die hohen Kosten von Kontrollaktivitäten muss jeder Aktionär für sich alleine tragen, die Erträge in Form höherer Aktienkurse werden unter allen Aktionären nach Massgabe ihrer Beteiligungsquoten verteilt. Daher sind entsprechende Kontrollanreize vor allem bei Blockaktionären, also institutionellen Investoren, vermögenden Privatpersonen (bzw. Familien), Hedge Fonds, etc., zu erwarten. Entsprechend umfangreich sind die Aktivitäten dieser Aktionärsgruppen untersucht worden.

Hinsichtlich des Einflusses von privaten Blockaktionären (z. B. Familien, Insidern) deuten die zahlreichen Studien tatsächlich auf eine positive Auswirkung auf die Unternehmensperformance hin. ${ }^{6}$ Hingegen gilt dies für den Einfluss von institutionellen Investoren nicht in dieser Klarheit, was möglicherweise auch mit den methodischen Problemen von Untersuchungen dieser Art zu tun hat. Dabei ist vor allem die Identifizierbarkeit von Ak-

5 So konstatiert Franck (2011) einen Trend zum shareholder empowerment, wobei man darauf hinweisen sollte, dass die dafür vorgetragenen Belege sich vor allem auf die USA und die Schweiz beziehen.

6 Vgl. Kaserer/Moldenhauer (2007) für einen Literaturüberblick, eine Diskussion der methodischen Probleme und eigene empirische Ergebnisse für den deutschen Kapitalmarkt. 
tionärsaktivitäten ein Problem. So muss man davon ausgehen, dass sich der Einfluss von institutionellen Investoren oft hinter den Kulissen abspielt und somit einer empirischen Untersuchung kaum zugänglich ist. Zudem sind Kontrollaktivitäten von Investoren mit langfristigem Anlagehorizont eher als evolutionärer Prozess zu sehen, bei dem es nicht auf einzelne spektakuläre Massnahmen ankommt. Entsprechend schwierig ist dann auch die statistische Identifikation von Kapitalmarktreaktionen. Daher verwundert es auch nicht, dass einschlägige Untersuchungen, etwa der Aktivitäten von Pensionsfonds oder anderer institutioneller Investoren, zu eher uneinheitlichen Ergebnissen kommen. ${ }^{7}$

Eine der wenigen Studien, die tatsächlich hinter die Kulissen solcher Kontrollaktivitäten schauen konnte, ist jene von Becht et al. (2010), in welcher aufgrund privat zur Verfügung gestellter Informationen der Erfolg von Kontrollaktivitäten des britischen Hermes Fund, der die Pensionsgelder von Britisch Telekom verwaltet, untersucht werden konnte. Die Autoren können zeigen, dass der Fonds nicht nur in vielfältiger Weise vor und hinter den Kulissen Einfluss auf geschäftspolitische Entscheidungen der Portfoliounternehmen nahm, sondern dabei auch überaus erfolgreich war. Im betrachteten Zeitraum von 1998 bis 2004 erzielte der Fonds fast 5\% Outperformance pro Jahr, wobei nach Schätzungen der Autoren an die $90 \%$ dieser Outperformance auf diese Kontrollaktivitäten zurückzuführen waren.

Wesentlich eindeutiger sind hingegen die Ergebnisse betreffend die Kapitalmarktreaktionen auf die Aktivitäten von Hedge Fonds, die vor allem in den 2000er Jahren stark zugenommen haben. Da die Aktivitäten dieser Fonds kurzfristig und auf bedeutende Einzelmassnahmen (z. B. Abstossen von Geschäftsbereichen, Aktienrückkäufe, Auswechslung des CEOs) ausgerichtet sind, lassen sich deren Ergebnisse sehr viel besser identifizieren. So zeigen Brav et al. (2008) in einer umfangreichen Analyse der Aktivitäten von Hedge Fonds über die Jahre 2001 bis 2006 in den USA, dass allein das Bekanntwerden des Einsteigens eines solchen Fonds zu einer hoch signifikanten positiven Kursreaktion in der Grössenordnung von $8 \%$ über einen Zweimonatszeitraum führt. Zudem finden sie keine Evidenz dafür, dass es im darauffolgenden Jahr zu einer Umkehr dieser Kursreaktion kommt. Eine Querschnittsanalyse zeigt, dass die Kursreaktionen dann am höchsten sind, wenn der Fonds eine Veränderung der strategischen Geschäftspolitik (z. B. durch einen Spin-off) anvisiert. Gibt er als Ziel hingegen lediglich die Veränderung der Finanzierungspolitik aus (z. B. durch eine hohe Einmalausschüttung), ist die positive Kursreaktion nicht mehr signifikant von Null verschieden. Interessant ist auch, dass das Gehaltsniveau des CEOs durch den Einstieg eines Hedge Fonds substantiell sinkt, während die operative Performance im Jahr nach dem Einstieg des Fonds unverändert bleibt.

Bestätigt werden diese Ergebnisse auch in anderen Studien, wie etwa jene von Klein/Zur (2009) und Achleitner et al. (2010). Letztere zeigen zudem, dass auch der Einstieg anderer aktiver Aktionärsgruppen, wie etwa Private Equity Fonds oder vermögender Privatpersonen (z. B. corporate raiders), zu ähnlichen Ergebnissen führt.

\section{Die Wirkung der Corporate-Governance-Regulierung}

Eine andere Möglichkeit der Frage nachzugehen, ob die Stärkung von Aktionärsrechten einen positiven Einfluss auf den Unternehmenswert hat, besteht darin, die Wirkung von Corporate-Governance-Regelungen zu untersuchen. Es gibt hierzu zwei äusserst umfang-

7 Ein guter Überblick zu US-amerikanischen Untersuchungen findet sich in Gillan/Starks (2007). 
reiche Literaturstränge. Der erste, der häufig unter dem Stichwort Law and Finance subsumiert wird, zeigt u. a. auf, dass der Grad an Investorenschutz einen Einfluss auf die Kapitalmarktentwicklung und damit auch auf den Wohlstand einer Volkswirtschaft hat. Einen Überblick zu dieser Literatur findet man in La Porta et al. (2000). Der zweite Strang beschäftigt sich mit der Frage, wie Corporate-Governance-Regelungen die Unternehmensbewertung beeinflussen. So zeigen Gompers et al. (2003), dass ein Portfolio von Unternehmen, deren Satzungsgestaltung besonders aktionärsfreundlich ist, über einen Zehnjahreszeitraum eine deutliche Outperformance erzielt gegenüber einem anderen Portfolio von Unternehmen, deren Satzungsgestaltung besonders unternehmensverwaltungsfreundlich ist.

Das Problem an diesen Studien ist allerdings, dass Corporate-Governance-Regelungen, soweit sie von einem Unternehmen überhaupt gestaltbar sind, das Ergebnis eines unternehmensinternen Optimierungsprozesses und damit letztlich endogen determiniert sind. Somit steht man vor einem Identifikationsproblem. Daher haben andere Studien versucht, die Aktienmarktreaktion auf eine exogene Variation von Corporate-Governance-Regelungen zu untersuchen. Ein frühes Beispiel ist die viel beachtete Studie von Szewczyk/Tsetsekos (1992) zu den Auswirkungen eines Gesetzes im Staate Pennsylvania, das im Wesentlichen auf die Beschränkung der Stimmrechtsausübung seitens eines potentiellen Übernehmers abzielte. Die Autoren kommen zu dem Ergebnis, dass die Aktien jener Unternehmen, die von dieser gesetzlichen Bestimmung besonders betroffen waren, einen abnormalen Kursrückgang von etwa $9 \%$ erlitten.

In dieselbe Richtung weist auch eine Untersuchung von Kaserer et al. (2000) zur Kurswirkung von Höchststimmrechten. In dieser Untersuchung wurden alle Fälle von Einführungen oder Aufhebungen von Höchststimmrechten bei börsennotierten deutschen Unternehmen gesammelt. Im +/- 5-Tageintervall um den Ankündigungszeitpunkt wird eine hoch signifikante Kursreaktion von $-2,1 \%$ bei der Einführung eines Höchststimmrechtes beziehungsweise $+2,0 \%$ bei dessen Aufhebung dokumentiert.

In einer jüngeren Studie stellen Larcker et al. (2011) fest, dass die mittlere Kursreaktion auf verschiedenen regulatorische Massnahmen, die in den USA im Bereich der Corporate Governance beschlossen wurden, tendenziell negativ war. Interessanterweise finden sie eine negative Reaktion auch bei solchen Massnahmen, die die Aktionärsrechte stärken, etwa im Zusammenhang mit einer stärkeren Einflussnahme auf die Stimmrechtsunterlagen des Unternehmens. Diese Befunde stehen auf dem ersten Blick in Widerspruch zu den oben genannten. Tatsächlich haben manche Autoren diese Befunde als Beleg für einen negativen Einfluss zu hoher Aktionärsrechte auf den Unternehmenswert interpretiert.

Wenngleich natürlich eine solche Interpretation zulässig ist, muss man darauf hinweisen, dass es auch eine ganz andere Erklärung für diese Ergebnisse geben kann. Da die Corporate Governance das Ergebniss eines unternehmensinternen Optimierungsprozess ist, kann staatliches Eingreifen schon allein deshalb eine negative Kursreaktion verursachen, weil damit eine neue Second-Best-Lösung gefunden werden muss, die in der Erwartung der Investoren schlechter ist als die alte Second-Best-Lösung. Das Ergebnis könnte somit auch einfach nur die Effektivität staatlichen Eingreifens in diesem Bereich in Frage stellen.

\section{Würdigung und Diskussion}

Die Kontrolle von börsennotierten Unternehmen ist das Ergebnis eines komplexen Prozesses, in welchem sämtliche Stakeholder (Aktionäre, Vorstände, Mitarbeiter, Aufsichtsräte) 
versuchen, ihre persönlichen Interessen bestmöglich zu vertreten. Da Aktionäre den wesentlichen Teil der mit allen Unternehmensentscheidungen verbundenen Risiken tragen, ist die Durchsetzung ihrer Vermögensinteressen von besonderer Bedeutung für eine effiziente Allokation der volkswirtschaftlichen Ersparnisse. Dies gilt umso mehr, als auf einem funktionierenden Kapitalmarkt die langfristigen Konsequenzen unternehmerischer Entscheidungen sich in den Aktienkursen widerspiegeln. Vermögensmaximierende Investoren haben daher die stärksten Anreize Fehlentscheidungen zu korrigieren. Somit sollte grundsätzlich eine kapitalmarktorientiere Kontrolle den wirksamsten Corporate-Governance-Mechanismus darstellen.

Es gibt viele Befunde, die diese Sichtweise stützen. Einige wenige sind in diesem Beitrag vorgetragen worden, viele andere nicht. Insbesondere sei hier auf Jensen (1993) verwiesen, der sich ausführlich mit dem Versagen interner Kontrollmechanismen beschäftigt. Gleichzeitig wurde in diesem Beitrag auch darauf verwiesen, dass die Untersuchungen zu den Wirkungen von Kontrollaktivitäten durch Aktionärsgruppen nicht ausschliesslich in eine Richtung zeigen. Insbesondere sind die Befunde zu den Aktivitäten institutioneller Investoren gemischt. Neben den methodischen Problemen, die hierfür ursächlich sein könnten, ist auch zu beachten, dass natürlich auch Investoren, ebenso wie Vorstände und Aufsichtsräte, Interessenskonflikten und Fehlanreizen unterliegen.

Dieser Aspekt ist im Hinblick auf institutionelle Investoren besonders hervorzuheben, weil ihre Bedeutung in den letzten Jahrzehnten stark gestiegen ist. So berichtet Zingales (2009), dass der Anteil der Marktkapitalisierung US-amerikanischer Gesellschaften, der von institutionellen Investoren gehalten wird, von unter 10\% im Jahre 1950 auf über $60 \%$ im Jahre 2005 gestiegen ist. Eine ähnliche Entwicklung lässt sich auch für Deutschland - und vermutlich den Rest Europas - konstatieren, wenngleich die Datenlage eine exakte Analyse nicht hergibt. Allerdings lässt sich aus den Zahlen der Deutschen Bundesbank ablesen, dass die von privaten Haushalten gehaltenen Investmentzertifikate im Jahre 1991 knapp 6\% des BIP betrugen, während sie im Jahr 2009 bei $23 \%$ des BIP lagen. Die direkten Aktienanlagen sind im gleichen Zeitraum bei rund $8 \%$ des BIP in etwa konstant geblieben. Wenngleich dieser Zuwachs in Investmentfondsanlagen sicherlich nicht ausschliesslich auf Aktienanlagen zurückgeht, dürften diese von dieser Entwicklung aber sicherlich nicht auszunehmen sein. Kaserer/Moldenhauer (2007) zeigen in Einklang damit, dass der meldepflichtige Stimmrechtsanteil von institutionellen Investoren im Zeitraum 1998 bis 2003 stark gestiegen ist.

Es mag viele Gründe für diese Entwicklung geben, zuvorderst natürlich die zunehmende Bedeutung privater Altersvorsorge und der damit einhergehende, auch staatlich geförderte, Zuwachs an von Lebensversicherungen und Pensionsfonds verwalteten Vermögensanlagen. Gerade vor dem Hintergrund der oben erwähnten empirischen Ergebnisse muss man sich daher nicht nur der Frage widmen, ob diese Aktionärsgruppen über hinreichende Einflussmöglichkeiten in den Unternehmen verfügen, sondern insbesondere auch diskutieren, ob sie die richtigen Anreize haben. Diese letztere Frage ist gewissermassen die Kehrseite der shareholder-empowerment-Medaille. Die Tatsache, dass in Deutschland, aber auch in anderen Ländern, Kapitalanlagegesellschaften immer noch zu einem erheblichen Teil vom Bankensektor kontrolliert werden, ist sicherlich ein beunruhigendes Faktum, weil es nahelegt, dass es hier zu Interessenskonflikten kommen kann.

Dabei bestehen diese Interessenkonflikte nicht nur deswegen, weil die hinter den Kapitalanlagegesellschaften stehenden Banken als Kreditgeber bei den Unternehmen engagiert 
sind. Sie ergeben sich auch, weil die variablen Vergütungen der Bankmanager über Investmentbankingaktivitäten in erheblichem Masse von Entscheidungen der Unternehmensverwaltungen abhängen können. Nicht zufällig schlägt daher Zingales (2009) vor, eine gesetzliche Trennung des Bankgeschäfts von Vermögensverwaltungsaktivitäten einzuführen. Ein weniger weitreichender Vorschlag wäre, dass Vermögensverwalter in sehr viel transparenterem Masse Auskunft über ihr Abstimmungsverhalten auf Hauptversammlungen geben müssen. Gleichzeitig könnte man auch daran denken, Abstimmungsergebnisse immer getrennt nach institutionellen Investoren und Streubesitzaktionären zu veröffentlichen.

Zusammenfassend bleibt aber festzuhalten, dass die notwendige Verbesserung der Unternehmenskontrolle nur über eine konsequente Weiterentwicklung der Aktionärsrechte in Verbindung mit einer Reduktion der Interessenskonflikte auf Seiten der institutionellen Investoren möglich ist. Die Hoffnung, die Unternehmenskontrolle würde sich dadurch verbessern, dass man den Unternehmensverwaltungen einen besseren Schutz vor den Aktionären gewährt, lässt sich jedenfalls durch empirische Erfahrungen nicht bekräftigen.

\section{Literaturhinweise}

Achleitner, A.-K./Betzer, A./Gider, J. (2010): Do Corporate Governance Motives Drive Hedge Fund and Private Equity Fund Activities?, in: European Financial Management, Jg. 16, S. 805-828.

Becht, M./Franks, J./Mayer, C./Rossi, S. (2010): Returns to Shareholder Activism: Evidence from a Clinical Study of the Hermes UK Focus Fund, in: Review of Financial Studies, Jg. 23, S. 3093-3123.

Brav, A.Jjiang, W./Partnoy, F./Thomas, R. (2008): Hedge Fund Activism, Corporate Governance, and Firm Performance, in: Journal of Finance, Jg. 63, S. 1729-1775.

Franck, E. (2011): Ist es an der Zeit, die Aktionärsrechte zu stärken?, in: Die Unternehmung, Jg. 65, S. 201-214.

Gillan, S.L./Starks, L.T. (2007): The Evolution of Shareholder Activism in the United States, in: Journal of Applied Corporate Finance, Jg. 19, S. 55-73.

Gompers, P./Ishi, J./Metrick, A. (2003): Corporate Governance and Equity Prices, in: Quarterly Journal of Economics, Jg. 118, S. 107-155.

Grossman, S./Hart, O. (1980): Takeover Bids, the Free-Rider Problem, and the Theory of the Corporation, in: Bell Journal of Economics, Jg. 11, S. 42-64.

Jensen, M.C. (1993): The Modern Industrial Revolution, Exit, and the Failure of Internal Control Systems, in: Journal of Finance, Jg. 48, S. 831-880.

Kaserer, C./Moldenhauer, B. (2007): Insider Ownership and Corporate Performance - New Evidence from Germany, in: Review of Managerial Science, Jg. 2, S. 1-35.

Kaserer, C./Rose, H./Wenger, E. (2000): Price Reactions of Anti-Takeover Actions - The Case of the German Voting Right Limitation, Unveröffentlichtes Manuskript, Universität Würzburg.

Klein, A./Zur, E. (2009): Entrepreneurial Shareholder Activism: Hedge Funds and Other Private Investors, in: Journal of Finance, Jg. 64, S. 187-229.

La Porta, R./Lopez-de-Silanes, F./Shleifer, A./Vishny. R. (2000): Investor Protection and Corporate Governance, in: Journal of Financial Economics, Jg. 58, S. 3-27.

Larcker, D.F./Ormazabal, G./Taylor, D.J. (2011): The Market Reaction to Corporate Governance Regulation, in: Journal of Financial Economics (im Erscheinen).

Roe, M. (2011): The Corporate Shaeholder's Vote and Its Political Economy, in Delaware and in Washington, August 2011, unter http://papers.ssrn.com/abstract_id=1884110. 
Szewczyk, S.H./Tsetsekos, G.P. (1992): State Intervention in the Market for Corporate Control, in: Journal of Financial Economics, Jg. 31, S. 3-23.

Zingales, L. (2009): The Future of Securities Regulation, Januar 2009, unter http://papers.ssrn.com/ abstract_id=1319648.

Christoph Kaserer, Dr., ist ordentlicher Professor für Finanzmanagement und Kapitalmärkte an der Technischen Universität München.

Anschrift: Technische Universität München, Lehrstuhl für Finanzmanagement und Kapitalmärkte, Arcisstr. 21, D-80290 München, Tel.: +49(0)89/289-25489, E-Mail: christoph.kaserer@wi.tum.de 